\title{
Application of scaled nucleation theory to metallic vapor condensation
}

\author{
Daniel M. Martinez \\ Frank T. Ferguson \\ Richard H. Heist \\ Fairfield University, rheist@fairfield.edu \\ Joseph A. Nuth III
}

Follow this and additional works at: https://digitalcommons.fairfield.edu/engineering-facultypubs Copyright 2001 American Institute of Physics

The final publisher PDF has been archived here with permission from the copyright holder.

\section{Peer Reviewed}

\section{Repository Citation}

Martinez, Daniel M.; Ferguson, Frank T.; Heist, Richard H.; and Nuth, Joseph A. III, "Application of scaled nucleation theory to metallic vapor condensation" (2001). Engineering Faculty Publications. 139. https://digitalcommons.fairfield.edu/engineering-facultypubs/139

\section{Published Citation}

Martınez, D. M., Ferguson, F. T., Heist, R. H., \& Nuth III, J. A. (2001). Application of scaled nucleation theory to metallic vapor condensation. The Journal of chemical physics, 115(1), 310-316. doi:10.1063/1.1378069.

This item has been accepted for inclusion in DigitalCommons@Fairfield by an authorized administrator of DigitalCommons@Fairfield. It is brought to you by DigitalCommons@Fairfield with permission from the rightsholder(s) and is protected by copyright and/or related rights. You are free to use this item in any way that is permitted by the copyright and related rights legislation that applies to your use. For other uses, you need to obtain permission from the rights-holder(s) directly, unless additional rights are indicated by a Creative Commons license in the record and/or on the work itself. For more information, please contact digitalcommons@fairfield.edu. 


\title{
Application of scaled nucleation theory to metallic vapor condensation
}

\author{
Daniel M. Martínez \\ Department of Chemical Engineering, University of Rochester, Rochester, New York 14627 \\ Frank T. Ferguson \\ Department of Chemistry, Catholic University of America, Washington, DC 20064 \\ Richard H. Heist \\ Nucleation Laboratory, Manhattan College, Riverdale, New York 10471 \\ Joseph A. Nuth III \\ Code 691, NASA-Goddard Space Flight Center, Greenbelt, Maryland 20771
}

(Received 28 February 2001; accepted 17 April 2001)

\begin{abstract}
In this paper we report that scaled nucleation theory (SNT) can describe moderately well the observed nucleation behavior of a significant number of refractory materials if a more appropriate value of a quantity commonly referred to as the excess surface entropy is used. With the availability of more reliable critical point and liquid property data, we are better able to calculate this quantity and we find that for refractory materials it can be as small as one half to one third the quantity traditionally used in its approximation. As a result of using more accurate values, we find considerably better agreement between SNT and experiment than what was originally determined. We also explain why using surface tension slope information to determine the excess surface entropy can lead to substantial errors in the SNT supersaturation prediction. (C) 2001 American Institute of Physics. [DOI: 10.1063/1.1378069]
\end{abstract}

\section{INTRODUCTION}

The condensation of supersaturated vapors has been of theoretical and experimental interest for many decades. Early theoretical work focused on the development of a description for predicting the onset of nucleation based on thermodynamic and kinetic arguments. The pioneering work of Volmer and Weber ${ }^{1}$ and the continued work of Becker and Döring ${ }^{2}$ and later Zeldovich ${ }^{3}$ led to the development of a description commonly referred to as the classical nucleation theory (CNT). CNT has had considerable success in predicting (usually qualitatively) the experimental supersaturations required to initiate homogeneous nucleation for a wide range of molecular fluids, despite the simplifying assumptions made in the original theory.

Over the past several decades, investigators have focused on correcting various inconsistencies that are believed to adversely affect CNT. Two major inconsistencies that have been addressed in the literature are CNT's failure to satisfy the law of mass action ${ }^{4}$ and an apparent error in the expression for the cluster size distribution for the limiting case of monomer "clusters." 5,6 These inconsistencies have been addressed in a revised theoretical model referred to as the internally consistent classical theory (ICCT), which has resulted in slightly better agreement with some experimental studies.

There have also been attempts to describe the nucleation behavior of various classes of compounds using a scaled form of the CNT nucleation rate equation. ${ }^{7-9}$ In 1986, Hale introduced one such scaled formalism, known as scaled nucleation theory (SNT), ${ }^{10}$ which utilizes critical point quantities to reduce the CNT rate equation into a material- independent form. Although this form is an approximation of CNT, agreement with experimental data for various molecular fluids is rather good. As a result of this success, SNT was subsequently applied to selected refractory (i.e., metallic and other high temperature materials) nucleation data ${ }^{11}$ with what appeared to be similar success. However, comparison of SNT with nucleation data from a wider range of refractory materials ${ }^{12}$ suggested that these materials, as a class, could not be described as accurately as the molecular fluids to which SNT was originally applied.

With the availability of more reliable critical point and liquid property data we find that this poor agreement is, in part, the result of using an overestimated approximation for the value of the excess surface entropy. We find that for refractory materials this quantity can be as small as one third the quantity originally used to conduct the SNT analysis. In light of this new information, we reexamine SNT within the context of an increasing amount of experimental refractory nucleation data in the literature. We also apply the modifications inherent in ICCT to SNT to determine if these modifications enhance the ability of SNT to describe experimental nucleation data. Finally, we address a concern for obtaining "reliable" excess surface entropy quantities based on the choice of the surface tension approximation made in SNT.

\section{REVIEW OF SCALED NUCLEATION THEORY}

The motivation for briefly reviewing SNT here is threefold. First, we demonstrate a more appropriate method for obtaining an expression for the SNT supersaturation in order to apply it to refractory material data. Second, we show how 
SNT needs to be modified to eliminate the inconsistencies addressed in ICCT. Finally, we develop a scaling approximation more suitable for describing the nucleation of refractory materials.

\section{A. Basic theory}

The CNT expression for the homogeneous nucleation rate $J$ in its general form can be expressed as

$$
J=J_{\mathrm{pre}}^{\mathrm{CNT}} \exp \left(-\frac{\Delta F^{\mathrm{CNT}}}{k_{B} T}\right) .
$$

Here, $J_{\mathrm{pre}}^{\mathrm{CNT}}$ is the preexponential rate term, $\Delta F^{\mathrm{CNT}}$ is the expression for the free energy associated with the formation of a liquid cluster from a supersaturated vapor, $k_{B}$ is the Boltzmann constant, and $T$ is the temperature.

The preexponential rate term in Eq. (1) can be expressed as

$$
J_{\mathrm{pre}}^{\mathrm{CNT}}=\left(\frac{2 \sigma}{\pi m \rho^{2}}\right)^{1 / 2}\left(\frac{S P_{\mathrm{sat}}}{k_{B} T}\right)^{2} .
$$

Here, $\sigma$ is the bulk liquid surface tension, $m$ is the molecular mass, $\rho$ is the molecular density, $S$ is the supersaturation ratio, and $P_{\text {sat }}$ is the saturation vapor pressure. Following Hale, ${ }^{10}$ an equivalent form for $J_{\text {pre }}^{\mathrm{CNT}}$ (denoted by a superscript SNT) is developed by introducing critical point properties (denoted by a subscript $c$ ) and the inverse thermal wavelength (denoted by $\lambda$ ) into Eq. (2) to yield the following expression:

$$
J_{\mathrm{pre}}^{\mathrm{SNT}}=J_{c} I\left(\frac{S P_{\mathrm{sat}}}{P_{c}}\right)^{2},
$$

where

$$
J_{c} \equiv\left[\left(\frac{P_{c}}{h}\right)\left(\frac{\lambda_{c}}{\rho_{c}}\right)\left(\frac{P_{c}}{k_{B} T_{c} \lambda_{c}}\right)\right]
$$

and

$$
I \equiv\left[\left(\frac{4}{3 \sqrt{\pi}}\right)^{1 / 3}(\theta)^{1 / 2}\left(\frac{T_{c}}{T}\right)^{3 / 2}\left(\frac{\rho_{c}}{\rho}\right)^{2 / 3}\right] .
$$

Here, $h$ is Planck's constant and $\theta$ is a grouping of terms common to nucleation theory given by

$$
\theta \equiv \frac{(36 \pi)^{1 / 3} \sigma}{\rho^{2 / 3} k_{B} T}
$$

The free energy term in Eq. (1) can be expressed as

$$
\Delta F^{\mathrm{CNT}}=\left(\frac{16 \pi}{3}\right)\left(\frac{\sigma}{\rho^{2 / 3}}\right)^{3}\left[\frac{1}{\left(k_{B} T \ln S\right)^{2}}\right] .
$$

Again, following Hale, the critical temperature is introduced into the free energy expression by approximating the surface tension with the following linear expression:

$$
\sigma=\sigma_{A}\left(T_{c}-T\right) .
$$

Here, $\sigma_{A}$ is a material-specific constant. Thus, by replacing the bulk surface tension with the approximation in Eq. (8), the exponential in Eq. (1) becomes

$$
-\frac{\Delta F^{\mathrm{CNT}}}{k_{B} T}=-\left(\frac{16 \pi}{3}\right) \Omega^{3}\left(\frac{T_{c}}{T}-1\right)^{3}\left[\frac{1}{(\ln S)^{2}}\right] .
$$

Here, $\Omega$ is a grouping of terms commonly referred to as the excess surface entropy, which contains bulk liquid information specific to the material of interest and is given by

$$
\Omega \equiv \frac{\sigma_{A}}{\rho^{2 / 3} k_{B}}
$$

Thus, with the information in Eqs. (1), (3), (9), and (10), $\ln S$ can then be expressed as

$$
\ln S=\Omega^{3 / 2} \beta_{0}^{\mathrm{CNT}}\left(\frac{T_{c}}{T}-1\right)^{3 / 2},
$$

where

$$
\beta_{0}^{\mathrm{CNT}} \equiv\left(\frac{16 \pi}{3}\right)^{1 / 3}\left[\ln \left(\frac{J_{\mathrm{pre}}^{\mathrm{SNT}}}{J}\right)\right]^{-\mathrm{i} / 2} .
$$

Equation (11) is equivalent to the supersaturation expression obtained from Hale's original scaled theory. ${ }^{10}$

It is noted that in the original derivation a similarity in behavior for $J_{\text {pre }}^{\text {SNT }}$ was observed for a variety of molecular fluids. It was also observed to be a relatively weak function of temperature, which allowed $\beta_{0}^{\mathrm{CNT}}$ in Eq. (12) to be approximated with a constant value (roughly equal to 0.53 ) for experimental nucleation rates of $1 \mathrm{~cm}^{-3} \mathrm{~s}^{-1}$. For rates other than unity, the following approximation was used:

$$
\ln S \sim 0.53 \Omega^{3 / 2}\left(1+\frac{\ln J}{2 \ln J_{c}}\right)\left(\frac{T_{c}}{T}-1\right)^{3 / 2} .
$$

Equation (13) was then compared to experimental nucleation rate data for a variety of molecular fluids and remarkable agreement was found. It was emphasized, however, that this approximate expression was found to be valid only for moderate experimental temperatures (i.e., when $\left.T_{c} / T-1<1.5\right)^{10}$

\section{B. Internally consistent scaled nucleation theory}

As mentioned earlier, ICCT was developed to correct certain inconsistencies apparent in CNT. The first correction deals with the fact that CNT fails to satisfy the law of mass action. Removing that inconsistency results in the introduction of the term $1 / S$ in the preexponential term of the CNT rate expression, and is referred to as the Courtney modification. ${ }^{4}$ The second correction deals with an error in the expression for the (classical) cluster size distribution when applied to the limiting case of the monomer. Although this is more of a bookkeeping inconsistency, it has been addressed by several authors who have proposed adding the term $\theta$ [see Eq. (6)] into the exponential form of the free energy. This is often referred to as the limiting consistency modification. 5,6 Thus, the resulting form for the ICCT rate expression can be written as 


$$
J=J^{\mathrm{CNT}} \cdot \frac{e^{\theta}}{S},
$$

which, when expanded as described in the previous section, gives

$$
\ln S=\Omega^{3 / 2} \beta_{0}^{\mathrm{ICCT}}\left(\frac{T_{c}}{T}-1\right)^{3 / 2},
$$

where

$$
\beta_{0}^{\mathrm{ICCT}} \equiv\left(\frac{16 \pi}{3}\right)^{1 / 2}\left[\ln \left(\frac{J_{\mathrm{pre}}^{\mathrm{SNT}}}{J S}\right)+(36 \pi)^{1 / 3} \Omega\left(\frac{T_{c}}{T}-1\right)\right]^{-1 / 2}
$$

in the SNT supersaturation expression. Here, $\beta_{0}^{\mathrm{ICCT}}$ is similar to $\beta_{0}^{\mathrm{CNT}}$ from Eq. (12) and is slightly smaller in value. Thus, the SNT prediction for $\ln S$ based on CNT will be slightly larger than for ICCT for any given system of interest.

\section{Determining "reliable" values for $\boldsymbol{\Omega}$}

In the past, ${ }^{10} \Omega$ has been evaluated by approximating $\sigma_{A}$ in Eq. (8) with $\sigma /\left(T_{c}-T\right)$. Since then, it has been suggested that a more "reliable" method for evaluating $\Omega$ is to approximate $\sigma_{A}$ with actual $d \sigma / d T$ information extracted from experimental surface tension data. ${ }^{13}$ However, others have raised the issue that by doing this a very different value for $\Omega$ can be obtained, resulting in a very different SNT prediction for the supersaturation. ${ }^{14-16}$ Compounding this issue is the fact that some experimental nucleation data appear to agree better with the original method while other data appear to agree better with this more "reliable" method. This has lead to uncertainty in deciding which method is most appropriate for comparing SNT to experimental nucleation data. In this work we have determined that although using $d \sigma / d T$ information from experimental surface tension data may at first appear to be appealing, it is inconsistent with the assumptions made in the SNT derivation and can, in fact, lead to substantial errors in the SNT supersaturation prediction.

Consider the plot shown in Fig. 1 of actual surface tension vs temperature data for liquid bismuth ${ }^{17}$ (denoted by the four closed circles). The surface tension decreases in an essentially linear fashion with temperature. If a line is used to pass through the experimental data and is extrapolated to the point of zero surface tension, as is shown by line $A$ in Fig. 1, it will intercept the temperature axis at an extrapolated point $T_{c}^{\text {ext }}$, which does not equal the actual critical temperature of bismuth (denoted by $T_{c}$ in Fig. 1). For most materials this is observed to be the case since, in general, in a region near the critical point, surface tension tends to deviate from linearity. ${ }^{18}$

An accurate fit to both the surface tension data and the actual critical temperature can be achieved by using a slightly more complex form for the surface tension. For example, the data can be fit to a power law expression similar (but slightly modified) to that suggested by Guggenheim ${ }^{19}$ given by

$$
\sigma=\sigma_{B}\left(T_{c}-T\right)^{\mu} .
$$

Here, $\sigma_{B}$ and $\mu$ are material-dependent constants that can be extracted from a linear least-squares fit to $\ln \sigma$ vs $\ln \left(T_{c}-T\right)$

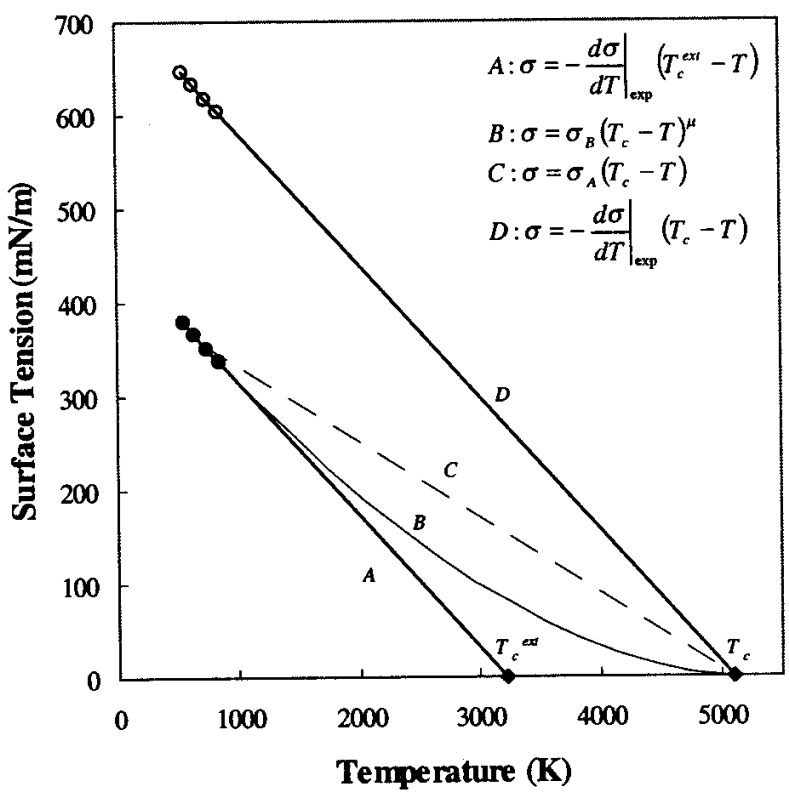

FIG. 1. Comparison of different approximations for the surface tension of liquid bismuth. The closed circles represent experimental data points from Ref. 17. The open circles represent the predicted surface tension values based on Eq. (18).

information. In the case of bismuth, $\mu$ is found to be 1.74 , which indicates that a rather large deviation from linearity exists as the critical point is approached. Surface tension data are not available for bismuth in the vicinity of the critical point, however it may be expected to behave in similar fashion to curve B in Fig. 1.

Recall from the SNT derivation that the approximate form used for the surface tension is that given in Eq. (8). In this form, the term $\sigma_{A}$ is in effect a two-point slope of the surface tension evaluated at the critical temperature (where $\sigma=0$ ) and at another available point. If several surface tension values are available, then in fact $\sigma_{A}$ is not constant and changes according to the choice of temperature (and necessarily, surface tension). A single surface tension value and the critical temperature are used to construct line $\mathrm{C}$ in Fig. 1. Since $T_{c}^{\mathrm{ext}} \neq T_{c}$, there is a discrepancy in the slope of this line and the slope of the experimental data. We note that using a single value of the surface tension in this linear approximation will cause surface tension values at other temperatures to be slightly higher or lower than what is found in the actual data.

Finally, consider the recommendation to use actual $d \sigma / d T$ information from experimental surface tension data as a better value for $\sigma_{A}$. The result is a linear approximation with an accurate experimental slope (denoted by a subscript exp) passing through the actual critical point, given by

$$
\sigma=-\left.\frac{d \sigma}{d T}\right|_{\exp }\left(T_{c}-T\right)
$$

A constant, experimentally obtained $d \sigma / d T$ value and the critical temperature are used to construct line D in Fig. 1. As can be seen, this expression gives an excellent approximation to the slope of the surface tension; however, it results in a far worse estimate for the actual surface tension of bismuth at 


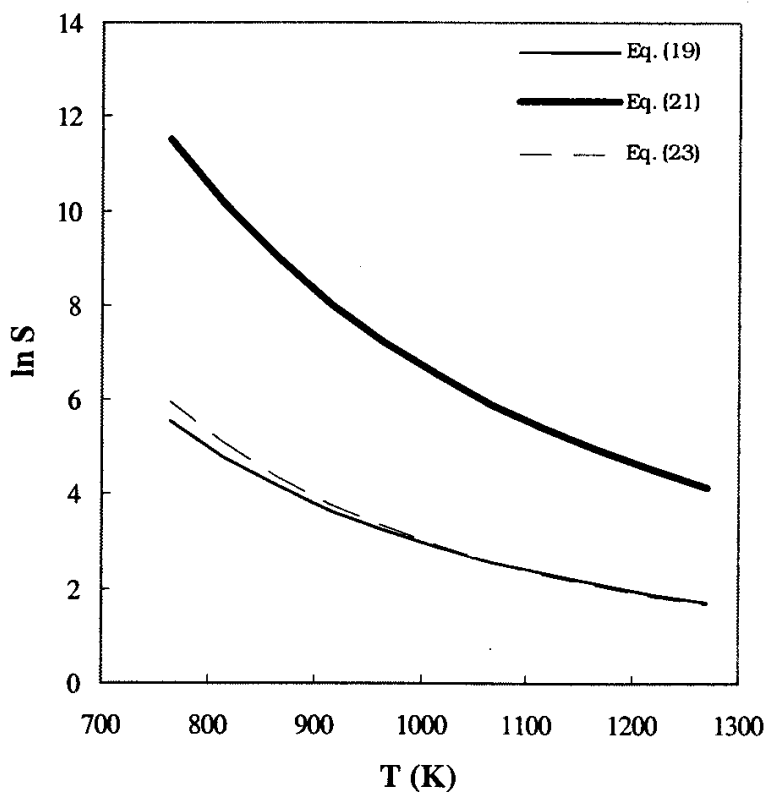

FIG. 2. Comparison of the predicted theoretical supersaturations for bismuth nucleation based on different approximations for the liquid surface tension in SNT. The similarity between Eqs. (19) and (23) indicates that Eq. (21) is an inappropriate method for use with SNT.

any given temperature. We note here that although using Eq. (18) results in a larger calculated surface tension value in the case of liquid bismuth, it may result in a smaller calculated value for other materials where $T_{c}^{\mathrm{ext}}$ is found to be greater than $T_{c}$.

Although surface tension values obtained using Eq. (18) can be substantially different than actual experimental data, it is possible that an accurate SNT supersaturation prediction depends on the use of a better estimate of $d \sigma / d T$ and not necessarily $\sigma$. In order to test this hypothesis we compare the SNT prediction for bismuth nucleation based on the two different "linear" methods for obtaining $\sigma_{A}$ [using Eqs. (8) and (18)] to the prediction based on the power law approximation in Eq. (17), as it is anticipated that this should give a better overall supersaturation prediction.

Rewriting the SNT predictions found in Eqs. (11) and (15) here to distinguish approximating $\sigma_{A}$ with $\sigma /\left(T_{c}-T\right)$ gives

$$
\ln S=\Omega_{1}^{3 / 2} \beta_{0}\left(\frac{T_{c}}{T}-1\right)^{3 / 2},
$$

where

$$
\Omega_{\mathrm{1}} \equiv \frac{\sigma}{\left(T_{c}-T\right) \rho^{2 / 3} k_{B}} .
$$

The predicted SNT supersaturation (CNT model only) as a function of temperature for bismuth nucleation based on Eq. (19) (represented by the thin solid curve) is shown in Fig. 2. The value used for $\Omega_{1}$ is taken at the midpoint of the temperature range.

Likewise, rewriting Eqs. (11) and (15) here to distinguish approximating $\sigma_{A}$ with an experimental $d \sigma / d T$ gives

$$
\ln S=\Omega_{2}^{3 / 2} \beta_{0}\left(\frac{T_{c}}{T}-1\right)^{3 / 2},
$$

where

$$
\Omega_{2} \equiv \frac{-d \sigma / d T}{\rho^{2 / 3} k_{B}} .
$$

The predicted SNT supersaturation based on Eq. (21) (represented by the thick solid curve) is also shown in Fig. 2. There is a large discrepancy between $\Omega_{1}$ and $\Omega_{2}$ for bismuth, resulting in a significant difference in the SNT prediction. We note that if no curvature were found in the actual surface tension data, $\Omega_{1}$ and $\Omega_{2}$ would give identical values.

In order to determine which supersaturation prediction is most accurate, we compare them to the power law form in Eq. (17) as it provides a better overall fit to actual surface tension data as well as the critical temperature and should therefore give the most reasonable SNT prediction. Carrying this form for the surface tension through the SNT derivation, we find that SNT must be adjusted accordingly to give

$$
\ln S=\Omega^{3 / 2} \beta_{0}\left[\frac{\left(T_{c}-T\right)^{\mu}}{T}\right]^{3 / 2},
$$

where $\beta_{0}$ remains the same for the CNT model and is altered for the ICCT model in the following manner:

$$
\begin{aligned}
\beta_{0}^{\mathrm{ICCT}} \equiv & \left(\frac{16 \pi}{3}\right)^{1 / 2}\left[\ln \left(\frac{J_{\mathrm{pre}}^{\mathrm{SNT}}}{J S}\right)\right. \\
& \left.+(36 \pi)^{1 / 3} \Omega\left(\frac{\left(T_{c}-T\right)^{\mu}}{T}\right)\right]^{-1 / 2} .
\end{aligned}
$$

We note that the $\Omega$ found in Eq. (23) is now defined as

$$
\Omega \equiv \frac{\sigma_{B}}{\rho^{2 / 3} k_{B}} .
$$

Figure 2 shows the predicted SNT supersaturation based on Eq. (23) (represented by the thin dashed curve). As can be seen, this improved surface tension fit offers only a modest improvement in predicting the supersaturation over the simpler, linear form used in Hale's original analysis where $\sigma_{A}$ was approximated by $\sigma /\left(T_{c}-T\right)$. This indicates that the importance in determining an accurate SNT prediction lies in using an accurate value for the surface tension and not so much its slope, thus the estimate for $\Omega$ in Eq. (20) is preferred over Eq. (22). Although this result has been demonstrated for a metallic system, a comparison to all classes of materials should yield similar results.

\section{D. $\mathbf{\Omega}$ for liquid metals}

Earlier investigators ${ }^{9,10}$ observed that Eq. (20) is identical in form to the Eötvös relationship ${ }^{20,21}$ evaluated on a molecular basis. Since this relationship was found to be successful in describing many classes of materials, and since there were tabulated values available for the Eötvös constant $^{9}$ (extrapolated to absolute zero and denoted by $k_{E, 0}$ ), Hale used these values as estimates for $\Omega$ in order to 
TABLE I. Values for the molecular excess surface entropy $\Omega$ for various liquid metals. Values for the molecular Eötvös constant are also shown here for comparison. Critical temperatures were obtained from Refs. 26-28. The terms $\sigma_{0}$ and $\rho_{0}$ represent the liquid surface tension and density extrapolated to $0 \mathrm{~K}$. These extrapolations were made in accordance with Ref. 9 from linear surface tension and density correlations obtained from Refs. 17 and 26.

\begin{tabular}{lrrrrc}
\hline \hline Material & $T_{c}(\mathrm{~K})$ & $\sigma_{0}(\mathrm{mN} / \mathrm{m})$ & $\rho_{0}\left(1 / \mathrm{m}^{3}\right)$ & $k_{E, 0}$ & $\Omega$ \\
\hline $\mathrm{Li}$ & 3482 & 475.12 & $4.90 E+28$ & 0.74 & 0.78 \\
$\mathrm{Na}$ & 2485 & 227.50 & $2.65 E+28$ & 0.75 & 0.78 \\
$\mathrm{~K}$ & 2280 & 133.88 & $1.40 E+28$ & 0.73 & 0.81 \\
$\mathrm{Rb}$ & 2017 & 102.57 & $1.13 E+28$ & 0.73 & 0.82 \\
$\mathrm{Cs}$ & 1938 & 84.81 & $9.09 E+28$ & 0.73 & 0.77 \\
$\mathrm{Mg}$ & 3783 & 719.67 & $4.46 E+28$ & 1.10 & 1.32 \\
$\mathrm{Ca}$ & 4876 & 470.65 & $2.46 E+28$ & 0.83 & 0.95 \\
$\mathrm{Mo}$ & 17193 & 3686.49 & $6.40 E+28$ & 0.97 & 0.76 \\
$\mathrm{~W}$ & 20399 & 3719.40 & $6.34 E+28$ & 0.83 & 0.73 \\
$\mathrm{Fe}$ & 10970 & 2393.90 & $8.64 E+28$ & 0.81 & 0.78 \\
$\mathrm{Ni}$ & 11152 & 2481.03 & $9.30 E+28$ & 0.79 & 0.75 \\
$\mathrm{Pt}$ & 10970 & 2491.76 & $7.62 E+28$ & 0.92 & 0.99 \\
$\mathrm{Cu}$ & 7868 & 1629.55 & $8.38 E+28$ & 0.78 & 0.85 \\
$\mathrm{Ag}$ & 6758 & 1102.00 & $5.82 E+28$ & 0.79 & 0.89 \\
$\mathrm{Au}$ & 8683 & 1752.76 & $5.84 E+28$ & 0.95 & 0.78 \\
$\mathrm{Zn}$ & 3275 & 899.76 & $6.75 E+28$ & 1.20 & 1.47 \\
$\mathrm{Cd}$ & 2886 & 653.19 & $4.67 E+28$ & 1.26 & 1.50 \\
$\mathrm{Hg}$ & 1748 & 546.57 & $4.28 E+28$ & 1.85 & 2.04 \\
$\mathrm{~Pb}$ & 5611 & 538.00 & $3.31 E+28$ & 0.67 & 0.70 \\
$\mathrm{Bi}$ & 5098 & 457.33 & $3.11 E+28$ & 0.66 & 0.61 \\
$\mathrm{Tl}$ & 4846 & 536.00 & $3.55 E+28$ & 0.74 & 0.78 \\
$\mathrm{Al}$ & 7748 & 1006.02 & $5.79 E+28$ & 0.63 & 0.66 \\
$\mathrm{Sn}$ & 7979 & 579.84 & $3.70 E+28$ & 0.47 & 0.51 \\
$\mathrm{Sb}$ & 5162 & 408.31 & $3.48 E+28$ & 0.54 & 0.60 \\
\hline \hline & & & & &
\end{tabular}

apply SNT to published experimental data. The values for $k_{E, 0}$ were observed to range from 1.5 (for associated species) to 2.1 (for "simpler" fluids) and using these values resulted in very good agreement between SNT and nucleation data for these types of fluids. SNT was subsequently compared to refractory nucleation data. However, since critical point and liquid property data were harder to find for the liquid metals, it was common practice to apply the values of $\Omega$ found for the simple and associated fluids to metallic species, as well. ${ }^{11,12,22,23}$ This typically resulted in poorer agreement between the theory and refractory experimental data and SNT was deemed unable to accurately describe refractory nucleation as a whole.

Based on the results of Hale's earlier work and our analysis above, we used Hale's original formalism employing Eq. (20) to actually evaluate $\Omega$ for the liquid metals. For many metals, it is found to be approximately 0.8 , which is consistent with other earlier observations involving these materials. ${ }^{24,25}$ Values for $\Omega$ as prescribed by Eq. (20) as well as values for $k_{E, 0}$ for various liquid metals are listed in Table I. Data for $\sigma(T)$ and $\rho(T)$ were obtained from published liquid density and surface tension data or correlations. ${ }^{17.26}$ For these calculations, when reliable critical point data were unavailable, values were estimated using boiling point and atomic radius data as prescribed by Aniya. ${ }^{26-28}$

As we have shown in Table I, $\Omega$ for the liquid metals can be roughly half (and in some instances a third) that for the associated liquids, which may indicate that at least part of the reason why SNT was unable to accurately predict experi- mental refractory data was due to the use of a significantly larger estimated excess surface entropy quantity. If this is true, then comparing SNT to experimental refractory nucleation data with these more accurate values for $\Omega$ should result in better overall agreement. We show this in Sec. III.

\section{COMPARISON WITH EXPERIMENTAL DATA}

Experimental devices amenable to metallic and refractory nucleation studies include the thermal diffusion cloud chamber (TDCC) ${ }^{29}$ the shock tube chamber (STC), ${ }^{30,31}$ and the gas evaporation chamber (GEC) ${ }^{32,33}$ The TDCC typically operates at experimental rates of $1 \mathrm{~cm}^{-3} \mathrm{~s}^{-1}$, while the STC and GEC typically operate at higher rates on the order of $10^{10} \mathrm{~cm}^{-3} \mathrm{~s}^{-1}$. For the most part, nucleation data from refractory nucleation investigations using these devices tend not to agree with conventional nucleation models. An exception to this observation are data from recent cesium nucleation studies utilizing a TDCC that showed reasonable agreement with CNT at the higher temperatures of the experiment. ${ }^{34} \mathrm{~A}$ comparison of SNT with nucleation rate data for lithium, ${ }^{23}$ sodium, ${ }^{35}$ bismuth, ${ }^{31}$ lead, ${ }^{31}$ iron, ${ }^{31}$ nickel ${ }^{36}$ magnesium, ${ }^{22}$ cesium, ${ }^{34}$ and mercury ${ }^{37}$ is reported below. In this analysis, the SNT supersaturation is scaled by the term $\Omega^{3 / 2}$ in order to interpret all of the data on one plot, as prescribed by Hale. ${ }^{10}$ Also, the TDCC experimental results are analyzed separately from the STC and GEC results since the difference in rate results in slightly different supersaturation predictions.

\section{A. STC and GEC rate data}

It was observed that for GEC and STC data for which the nucleation rate was estimated to be approximately equal to $10^{10} \mathrm{~cm}^{-3} \mathrm{~s}^{-1}$ the value of $\beta_{0}$ was roughly constant over the temperature range of interest and equal to 0.75 based on CNT or 0.65 based on ICCT. Using the values for $\Omega$ obtained above [using Eq. (20) with a value of $\sigma$ and $\rho$ at the midpoint of the data] and the (constant) values of $\beta_{0}$ found here, reasonable agreement with SNT is found for several experimental datasets while poor agreement is found for others as can be seen in Fig. 3. It is observed that significant deviations occur for the lithium, magnesium, and bismuth data. Recent results for sodium vapor nucleation ${ }^{35}$ using the GEC suggest to us that the deviation in the lithium data may be the result of an inappropriate correction factor applied to the raw data during analysis. We are currently reexamining our experimental data in order to verify this hypothesis. The deviation in the magnesium and bismuth data is interesting since the value of $\Omega$ obtained for these materials is either significantly larger, i.e., 1.3 for $\mathrm{Mg}$, or moderately smaller, i.e., 0.6 for $\mathrm{Bi}$, than that found for most of the other liquid metals in this analysis, i.e., 0.8 (see Table I). We note that using a value of 0.8 would result in better agreement with the SNT supersaturation prediction for both the $\mathrm{Mg}$ and the Bi data. Uncertainties in values of $T_{c}$ do not appear to be able to account for these discrepancies. 


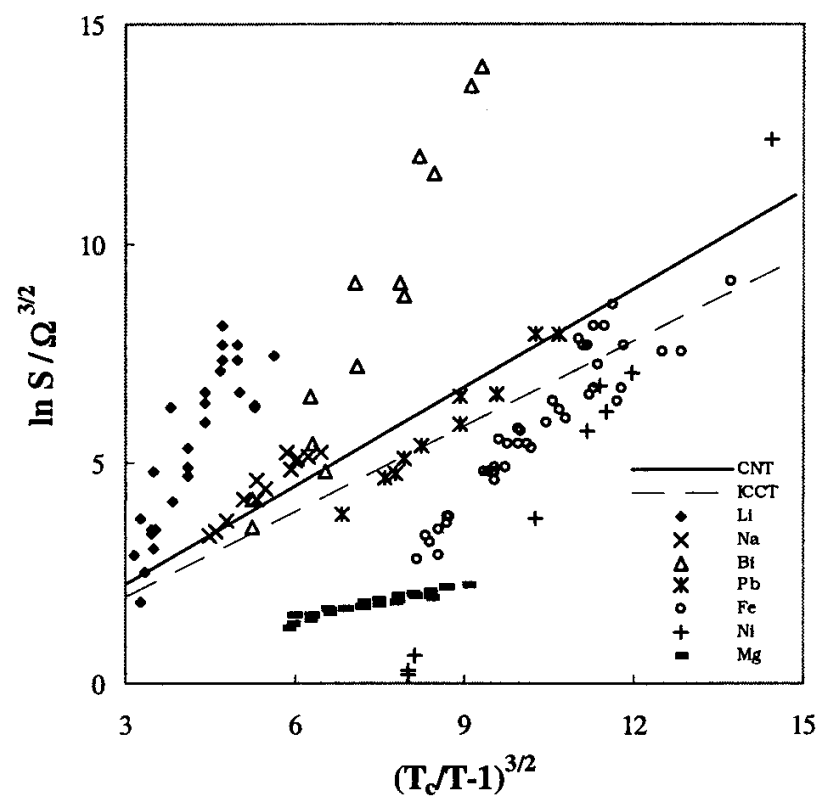

FIG. 3. Comparison of metallic nucleation data (for $J \sim 10^{10} \mathrm{~cm}^{-3} \mathrm{~s}^{-1}$ ) with the SNT supersaturation expressions given in Eqs. (11) and (15). The values for $\beta_{0}$ in this analysis are approximated with a constant and are 0.75 and 0.65 for CNT and ICCT models, respectively. Equations (11) and (15) are scaled by $\Omega^{3 / 2}$, which varies for each metal. The solid and dashed lines represent the SNT approximation based on CNT and ICCT models, respectively. Experimental data were obtained from Refs. 22, 23, 31, 35, and 36. The number of data points shown for lithium and magnesium is reduced for clarity; however, the general trend and spread in the data are preserved.

\section{B. TDCC critical supersaturation data}

It was observed that for TDCC critical supersaturation data for mercury vapor the value of $\beta_{0}$ was nearly constant and equal to 0.49 based on CNT or 0.40 based on ICCT. However, for TDCC critical supersaturation data for cesium vapor, $\beta_{0}$ changed significantly over the temperature range of the study and needed to be accounted for. Thus, the expressions for $\beta_{0}$ in Eqs. (12) and (16) were used with actual experimental data along with Eqs. (11) and (15) for the scaled supersaturation and Eq. (20) for $\Omega$ to determine the SNT predictions for comparison with both the cesium and mercury data. Figure 4 shows experimental homogeneous vapor-to-liquid data for $\ln S / \Omega^{3 / 2}$ for both cesium and mercury. Overall agreement is reasonable for cesium with the SNT prediction based on the CNT model and we see that SNT predicts a curvature similar to that observed in the lower temperatures of the cesium nucleation data. The ICCT model is not shown in Fig. 4 since it tends to underpredict what is observed in experiment (interestingly, though, the ICCT curve is parallel to the data). Mercury, on the other hand, shows poorer agreement with the SNT prediction. It is interesting to note that the value of $\Omega$ for mercury is approximately 2.0, which like magnesium, is large compared to most of the liquid metals (see Table I) and may indicate why agreement is so poor.

\section{Comment on using larger $\mathbf{\Omega}$ values}

We remark here that if we follow previous recommendations and use a value for $\Omega$ of 2.0 for all nine metallic species analyzed in this work, we find that SNT greatly over-

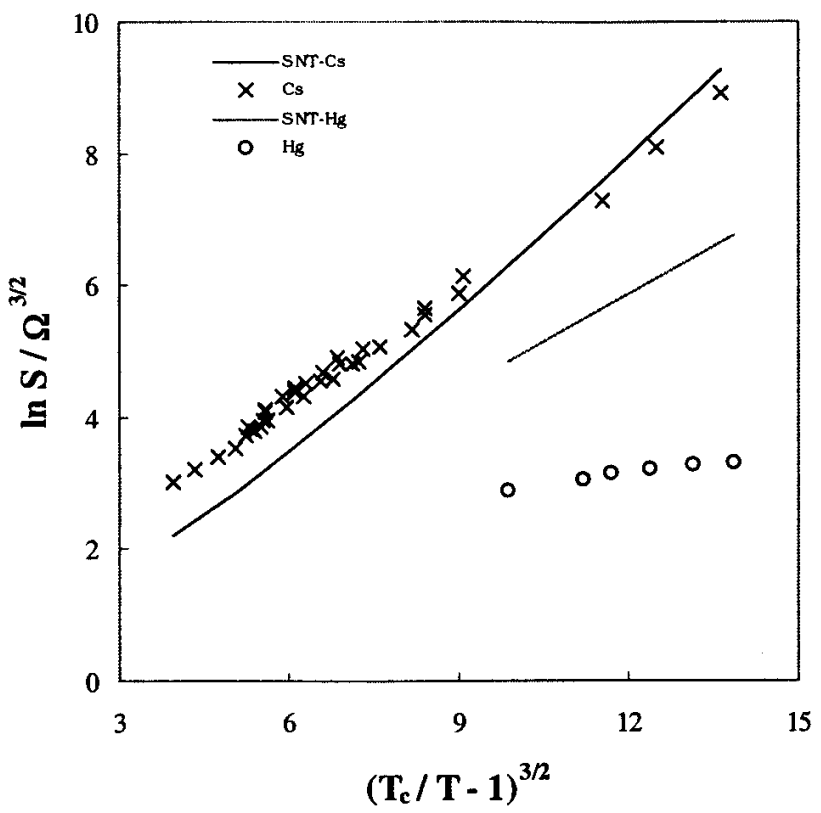

FIG. 4. Comparison of metallic nucleation data (for $J \sim 1 \mathrm{~cm}^{-3} \mathrm{~s}^{-1}$ ) with the scaled supersaturation expression given in Eq. (11). The value for $\beta_{0}$ was calculated directly for cesium using Eq. (12) while a constant of 0.49 was used for comparison with mercury. The solid black and gray curves represent SNT based on CNT model. Experimental data were obtained from Refs. 34 and 37.

predicts what is observed in experiment. To demonstrate, we reanalyze the cesium critical supersaturation data with an approximated $\Omega$ of 2.0 and plot the results accordingly in Fig. 5. Using such a large value for $\Omega$ (relative to what we now calculate) results in a downward shift in both the theoretical and experimental curves, however the shift in the experimental curve is much more dramatic. This disparity is also found when the STC and GEC data are reanalyzed in

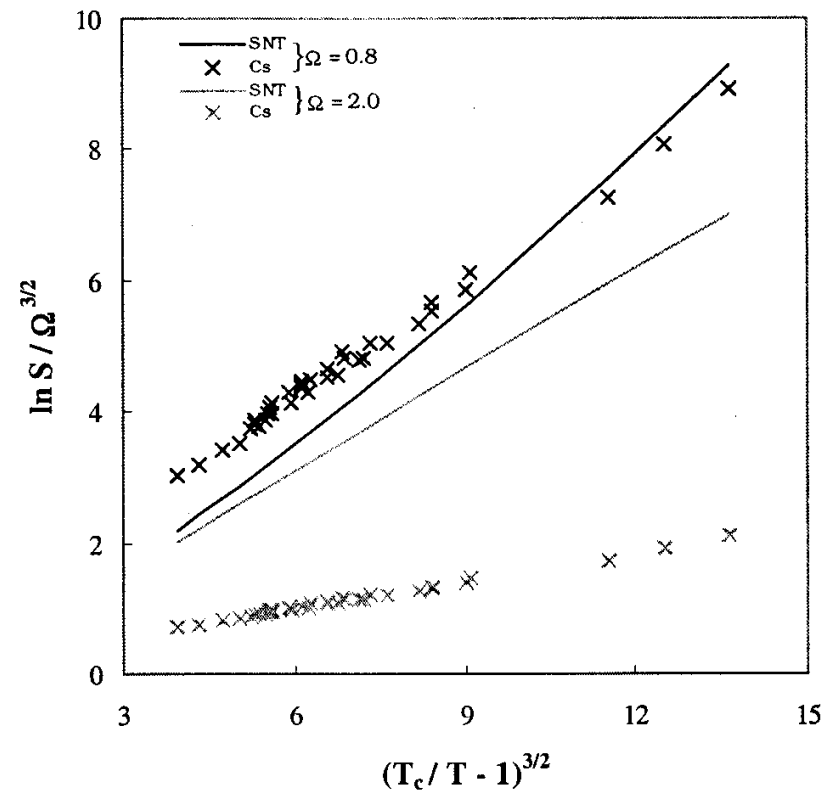

FIG. 5. Comparison of the SNT scaled supersaturation with cesium experimental data based on a value for $\Omega$ of 0.8 and 2.0 , respectively. The larger $\Omega$ value results in very poor agreement between theory and experiment. The solid black and gray curves represent the SNT prediction based on the CNT model. Experimental data were obtained from Ref. 34. 
this manner. Using more realistic values such as the ones tabulated in Table I results in better overall agreement, however discrepancies between SNT and some of the datasets may indicate that other problems exist, such as inappropriate assumptions in the underlying theory (i.e., capillarity) or, potentially, incorrect experimental data. It is clear that more experimental studies need to be carried out in order to prove (or disprove) these hypotheses.

\section{CONCLUSIONS AND REMARKS}

In 1986, Hale published an approximate scaling law based on a reduced form of the CNT rate expression and compared it to homogeneous nucleation data for various molecular liquids with remarkable success. However, later comparisons of SNT with a wide range of refractory nucleation data resulted in generally poor agreement. In this paper we report that the reason for such poor agreement appears to be, at least in part, the result of using an overestimated approximation of the value for the excess surface entropy (i.e., $\Omega$ ) for liquid metals. While the value for $\Omega$ is roughly 2.1 for simple liquids and 1.5 for associated liquids, it is reported here to be approximately 0.8 for most metallic liquids. This observation has a significant impact on how well SNT describes homogeneous nucleation data of refractory vapors, since the perceived value of $\sim 2.0$ typically resulted in poor agreement. These smaller quantities were calculated by approximating $\sigma_{A}$ with $\sigma /\left(T_{c}-T\right)$ since it was determined that using actual $d \sigma / d T$ information is unreliable and inconsistent with the assumptions made in SNT.

As part of our analysis, a version of SNT consistent with ICCT was derived and compared with existing nucleation data for refractory materials. Based on the quality of the experimental data available for the nucleation of refractory materials, there is no clear advantage yet to choosing either CNT or ICCT to form the scaled supersaturation expressions used in SNT. One form of SNT works reasonably well describing the nucleation of some refractory materials while the other form works better for others.

Refractory materials, as a class, appear to behave differently than the simple fluids studied in Hale's original work as is evident by their unusually low $\Omega$ values for many of these materials and relatively large values for a few of them, i.e., $\mathrm{Hg}$ and $\mathrm{Mg}$. Clearly, the use of bulk liquid properties to describe a process involving small metallic clusters is problematic. Even though several of the data sets shown in Figs. 3 and 4 appear to be in reasonable agreement with SNT, it is clear that there is a serious need for more and better nucleation data for refractory materials.

\section{ACKNOWLEDGMENT}

This work was supported in part by the National Aeronautics and Space Administration's Graduate Student Researchers Program Grant No. NGT-5-104.

${ }^{1}$ M. Volmer and A. Weber, Z. Phys. Chem. (Leipzig) 119, 277 (1925).

${ }^{2}$ R. Becker and W. Döring, Ann. Phys. (Leipzig) 24, 719 (1935).

${ }^{3}$ J. Zeldovich, J. Exp. Theor. Phys. 12, 525 (1942).

${ }^{4}$ W. G. Courtney, J. Chem. Phys. 35, 2249 (1961).

${ }_{5}^{5}$ S. L. Gershick and C.-P. Chiu, J. Chem. Phys. 93, 1273 (1990).

${ }^{6}$ S. L. Gershick, J. Chem. Phys. 94, 826 (1991).

${ }^{7}$ B. J. C. Wu, P. P. Wegner, and G. D. Stein, J. Chem. Phys. 68, 308 (1978).

${ }^{8}$ R. McGraw, J. Chem. Phys. 75, 5514 (1981).

${ }^{9}$ D. H. Rasmussen and S. V. Babu, Chem. Phys. Lett. 108, 449 (1984).

${ }^{10}$ B. Hale, Phys. Rev. A 33, 4156 (1986).

${ }^{11}$ B. Hale, P. Kemper, and J. A. Nuth III, J. Chem. Phys. 91, 4314 (1989).

${ }^{12}$ J. A. Nuth III and F. T. Ferguson, in Nucleation and Crystallization in Liquids and Glasses, edited by M. C. Weinberg (American Ceramic Society, Westerville, OH, 1993), Vol. 30, p. 23.

${ }^{13}$ B. N. Hale and B. Kelley, Chem. Phys. Lett. 189, 100 (1992).

${ }^{14}$ D. Wright and M. S. El-Shall, Chem. Phys. Lett. 189, 103 (1992).

${ }^{15}$ D. Wright, R. Caldwell, C. Moxely, and M. S. El-Shall, J. Chem. Phys. 98, 3356 (1993).

${ }^{16}$ D. Kane and M. S. El-Shall, J. Chem. Phys. 105, 7617 (1996).

${ }^{17}$ CRC Handbook of Chemistry and Physics, 63rd ed., edited by R. C. Weast (CRC, Boca Raton, FL, 1982).

${ }^{18}$ A. W. Adamson, Physical Chemistry of Surfaces, 4 th ed. (Wiley, New York, 1982).

${ }^{19}$ E. A. Guggenheim, J. Chem. Phys. 13, 7 (1945).

${ }^{20} \mathrm{R}$. Eötvös, Wied. Ann. Phys. 27, 456 (1886).

${ }^{21}$ J. E. Lennard-Jones and J. Corner, Trans. Faraday Soc. 36, 1156 (1940).

${ }^{22}$ F. T. Ferguson, J. A. Nuth III, and L. U. Lilleleht, J. Chem. Phys. 104, 3205 (1996).

${ }^{23}$ F. T. Ferguson and J. A. Nuth III, J. Chem. Phys. 113, 4093 (2000).

${ }^{24}$ A. V. Grosse, J. Inorg. Nucl. Chem. 24, 147 (1962).

${ }^{25}$ S. Blairs and U. Joasoo, J. Colloid Interface Sci. 79, 373 (1981).

${ }^{26}$ CRC Handbook of Chemistry and Physics, 78th ed. (CRC, New York, 1997).

${ }^{27}$ F. Hensel, J. Phys.: Condens. Matter 2, SA33 (1990).

${ }^{28}$ M. Aniya, Physica B 239, 144 (1997).

${ }^{29}$ G.-S. Cha, H. Uchtmann, J. A. Fisk, and J. L. Katz, J. Chem. Phys. 101, 459 (1994).

${ }^{30}$ J. R. Stephens and S. H. Bauer, Proceedings of the 13th International Symposium on Shock Tubes and Waves (State University of New York Press, Albany, NY, 1982).

${ }^{31}$ D. J. Frurip and S. H. Bauer, J. Phys. Chem. 81, 1001 (1977).

${ }^{32}$ J. A. Nuth III, K. A. Donnelly, B. Donn, and L. U. Lilleleht, J. Chem. Phys. 85, 1116 (1994).

${ }^{33}$ J. A. Nuth III and B. Donn, J. Chem. Phys. 77, 2639 (1982).

${ }^{34}$ M. M. Rudek, J. L. Katz, and H. Uchtmann, J. Chem. Phys. 110, 11505 (1999).

${ }^{35}$ F. T. Ferguson, D. M. Martínez, and J. A. Nuth III (unpublished).

${ }^{36}$ J. Steinwandel and J. Hoeschele, J. Chem. Phys. 85, 6765 (1986).

${ }^{37}$ H. Uchtmann, K. Rademann, and F. Hensel, Ann. Phys. (Leipzig) 48, 207 (1991). 